\title{
PARÂMETROS BIOQUÍMICOS DE REFERÊNCIA DE RATOS WISTAR DE DIFERENTES FAIXAS DE PESO, PROVENIENTES DO BIOTÉRIO DAS FACULDADES NOVA ESPERANÇA - PB
}

\author{
João Vinícius Barbosa Roberto' \\ Claudionor Soares do Nascimento Júnior " \\ Elisa Serra Alvim de Souza ${ }^{\text {II }}$ \\ Ravena Alves Martins ${ }^{\text {III }}$ \\ Edvaldo Ribeiro de Araújo IV \\ Márcia Maria Araújo de Barros IV
}

\begin{abstract}
RESUMO
Os roedores são sistemas biológicos sensíveis a fatores externos e internos, de maneira que é evidente a existência de variações intraespécies nos resultados experimentais, sendo certamente produtos de diferenças ambientais, do manuseio e/ou metodologia adotados. Portanto, é imprescindível se estabelecer valores de referência próprios para cada biotério ou laboratório, bem como divulgá-los. O estudo objetiva estabelecer os valores de referência para parâmetros bioquímicos de ratos Wistar não-tratados, provenientes do Biotério João Bezerra de Lima, das Faculdades Nova Esperança, Paraíba, Brasil. Foram utilizadas amostras sanguíneas de 83 ratos Wistar, machos e fêmeas, adultos, saudáveis, de diferentes gerações, pesando entre 200 a $500 \mathrm{~g}$ nos machos e 150 a $300 \mathrm{~g}$ nas fêmeas, provenientes do Biotério João Bezerra de Lima, das Faculdades Nova Esperança - PB. Foram mensurados os parâmetros bioquímicos glicose, triglicerídeos, colesterol total, HDL, as enzimas AST e ALT e uréia e creatinina. O Estudo foi avaliado pelo CEUA FACENE/FAMENE e julgado aprovado, sob o protocolo $n^{\circ}$ 0051.2017.1. As médias de alguns dos parâmetros bioquímicos registradas são as seguintes: Glicose: (Machos de 200 a 370 g - 245,13 mg/dL1; machos 380 a $500 \mathrm{~g}$ - 212,39 mg/dL-1; Fêmeas de 150 a 230g - 170,38 mg/dL-1; Fêmeas de 230 a 300 g - 166,07 mg/dL-1); Colesterol total: (Machos de 200 a 370 g -54,69 mg/dL-1; machos 380 a 500 g - 44,96 mg/dL-1; Fêmeas de 150 a $230 \mathrm{~g}-69,69$ mg/dL-1; Fêmeas de 230 a 300 g -71,85 mg/dL-1); Uréia:( Machos de 200 a 370 g -49,57 mg/dL-1; machos 380 a 500 g - 49,97 mg/dL-1; Fêmeas de 150 a 230g - 40,20 mg/dL-1; Fêmeas de 230 a $300 \mathrm{~g}$ $-40,38 \mathrm{mg} / \mathrm{dL}-1)$. Os resultados caracterizam as condições fisiológicas dos animais mantidos no biotério das Faculdades Nova Esperança - PB. Vários fatores intrínsecos e extrínsecos podem promover a variação dos parâmetros, assim, cada centro de pesquisa deve ter seus valores fisiológicos estabelecidos.
\end{abstract}

\section{PALAVRAS-CHAVE}

Bioquímica. Biotério. Ratos. Valores de referência. Wistar.

\section{INTRODUÇÃO}

A experimentação animal na pesquisa científica tem uma importante participação na descoberta de tratamento e medidas profiláticas para as diversas patologias que atingem os humanos. Animais de várias espécies têm sido utilizados nos últimos tempos, entretanto, os roedores, como o camundongo e os ratos, são os mais intensamente utilizados e profundamente conhecidos cientificamente 1 .

I. Doutor em Medicina Veterinária pela Universidade Federal de Campina Grande. Professor das Faculdades Nova Esperança - Rua Bancário Waldemar de M. Aciioly, 317; CEP 58051-420, João Pessoa-PB. Fone: 839 91368916, E-mail: profvinicius@facene.com.br ;

II Discente do curso de Farmácia das Faculdades Nova Esperança - João Pessoa-PB; Aluno do Programa de Iniciação Científica - PRICEA - FACENE/FAMENE.

III Discentes do curso de Medicina das Faculdades Nova Esperança - João Pessoa-PB; Alunas do Programa de Iniciação Científica - PRICEA - FACENE/FAMENE.

IV Funcionários, Técnicos das Faculdades Nova Esperança - João Pessoa-PB. 
Os roedores apresentam características que fizeram com que eles tivessem preferência em relação aos outros animais. Eles apresentam tamanho reduzido, ciclo reprodutivo curto, prole numerosa, precocidade, semelhança fisiológica com o organismo humano, nutrição variada, fácil domesticação, adaptação a ambientes variados e sociabilidade ${ }^{2}$.

Os ratos, depois dos camundongos, são os animais de laboratório mais comumente utilizados em pesquisas científicas, representando cerca de $20 \%$ do número total de animais usados nestas atividades, segundo Harkness e Wagner 3. De acordo com Fagundes e Taha4 e Cooper e Johnson5, a maior parte da pesquisa na área básica é empreendida nos animais de pequeno porte (camundongo, rato, hamster, cobaio, ou o gerbil) e compreendem quase $90 \%$ do total das espécies utilizadas nos laboratórios.

Nos experimentos e pesquisas científicas, para se ter uma correta avaliação e interpretação das modificações induzidas pelos procedimentos experimentais, é necessário o conhecimento, o controle e a correta mensuração dos diversos parâmetros fisiológicos dos animais utilizados nas pesquisas. Porém, esses parâmetros podem apresentar variações relacionadas com gênero, idade, peso, dieta, linhagem, genótipo, manuseio, ambiente, entre outros fatores.

Além disso, os animais ainda podem se comportar de maneiras muito distintas, dependendo das condições a que

\section{MATERIAL E MÉTODOS}

O experimento foi conduzido no Biotério João Bezerra de Lima, pertencente as Faculdades Nova Esperança - PB. Foram utilizadas amostras sanguíneas de 83 animais da espécie Rattus novergicus, linhagem Wistar, entre machos e fêmeas, sendo 44 machos e 39 fêmeas, pesando entre 200 a $500 \mathrm{~g}$ nos machos e 150 a $300 \mathrm{~g}$ nas fêmeas.

Os animais foram alojados na sala são submetidos, sendo também passíveis de sofrerem influência de fatores ecológicos, característicos de cada região do planeta 6 . Os animais experimentais não se comportam da mesma maneira nas diferentes condições de criação.

O conhecimento e a padronização dos parâmetros fisiológicos, para cada espécie, é importante, uma vez que serve como referência para a avaliação do estado de higidez, de modificações induzidas por processos patológicos e para a avaliação dos resultados obtidos nos procedimentos experimentais7. A literatura descreve alguns trabalhos com valores de referência de parâmetros bioquímicos e hematológicos de animais não tratados $8,9,10$ e neles, observa-se que em alguns, há grandes variações, o que confirma as variações intraespécie, decorrentes de diferenças geográficas e de manuseio ${ }^{11}$.

Nesse contexto, torna-se imprescindível que cada instituição de pesquisa, laboratórios e biotérios, estabeleçam um conjunto próprio de dados fisiológicos dos animais normais, que irão servir como referência para as pesquisas e experimentos da instituição e de outros locais.

Desta forma, objetivou-se, com o trabalho, determinar os valores de referência para os parâmetros bioquímicos de ratos não-tratados, provenientes do Biotério João Bezerra de Lima, das Faculdades Nova Esperança- $\mathrm{PB}$.

de experimentação do Biotério João Bezerra de Lima, cuja temperatura ambiente e umidade relativa do ar são controladas e apresentaram valores de $23^{\circ} \mathrm{C} \pm 2^{\circ} \mathrm{C}$ e $40 \% \pm$ $10 \%$, respectivamente. Foram acondicionados em caixas de polipropileno, com cama de maravalha, submetidos a um ciclo claro e escuro de 12 horas, comida e água ad libitum. A alimentação foi constituída de ração balanceada padronizada para roedores. 
O Estudo foi avaliado pelo CEUA FACENE/FAMENE e julgado aprovado, sob o protocolo $n^{\circ}$ 0051.2017.1.

Os animais foram submetidos a 12 horas de jejum antes da coleta, anestesiados com xilasina a $2 \%$ (10 mg.Kg-1) e quetamina a 10\% (100 mg.Kg -1), através da via intraperitoneal. Após a anestesia e a confirmação desta pelos testes de reflexos, foram fixados em mesa cirúrgica. O abdômen foi aberto até a altura do diafragma por uma incisão longitudinal central e duas incisões laterais para exposição da veia cava inferior infra-hepática, de onde foi subtraído aproximadamente $4 \mathrm{~mL}$ de sangue (exsanguinação). Após a coagulação do sangue em tubos secos, os tubos foram centrifugados,

\section{RESULTADOS E DISCUSSÃO}

Os parâmetros bioquímicos de ratos wistar não tratados, provenientes do Biotério João Bezerra durante 10 minutos a 3500 rpm (rotações por minuto), para a separação do soro.

Os parâmetros bioquímicos foram obtidos por meio de um analisador bioquímico Prietest Touch ${ }^{\circledR}$, de acordo com as orientações do fabricante. Foram mensurados os seguintes parâmetros: glicose, triglicerídeos, colesterol total, HDL, as enzimas aspartato aminotransferase e alanina aminotransferase, ureia e creatinina.

Os resultados numéricos foram expressos em média aritmética e desvio padrão ( \pm erro padrão). Os animais foram divididos em grupos por sexo (masculino e feminino), sendo subdivididos em subgrupos por faixa de peso (machos de 200 a 370 g; machos de 380 a 500 g; fêmeas de 150 a $230 \mathrm{~g}$ e fêmeas de 230 a $300 \mathrm{~g}$ ).

de Lima das Faculdades Nova Esperança - PB, estão apresentados na Tabela 1.

Tabela 1 - Valores médios e desvio padrão (DP) dos parâmetros bioquímicos de ratos wistar não tratados, de ambos os gêneros, por diferentes faixas de peso.

\begin{tabular}{|c|c|c|c|c|}
\hline \multirow[b]{2}{*}{$\begin{array}{l}\text { Parâmetros Bioquímicos } \\
\text { (Unid.) }\end{array}$} & \multicolumn{4}{|c|}{$\begin{array}{c}\text { Sexo } \\
\text { Média +DP }\end{array}$} \\
\hline & $\begin{array}{c}\text { Machos } \\
(200 \text { a } 370 \mathrm{~g})\end{array}$ & $\begin{array}{c}\text { Machos } \\
(200 \text { a } 370 \mathrm{~g})\end{array}$ & $\begin{array}{c}\text { Fêmeas } \\
(150 \text { a 230 g) }\end{array}$ & $\begin{array}{c}\text { Fêmeas } \\
(230 \text { a } 300 \mathrm{~g})\end{array}$ \\
\hline Glicose (mg/dL $\left.{ }^{-1}\right)$ & $245,13 \pm 31,00$ & $212,39 \pm 70,75$ & $170,38 \pm 59,77$ & $166,07 \pm 83,02$ \\
\hline Triglicerídeos $\left(\mathrm{mg} / \mathrm{dl}^{-1}\right)$ & $64,49 \pm 27,30$ & $46,73 \pm 19,41$ & $78,68 \pm 43,99$ & $95,10 \pm 73,17$ \\
\hline Colesterol total $\left(\mathrm{mg} / \mathrm{dl}^{-1}\right)$ & $54,69 \pm 22,15$ & $44,96 \pm 12,61$ & $69,69 \pm 25,90$ & $71,85 \pm 30,66$ \\
\hline $\mathrm{HDL}\left(\mathrm{mg} / \mathrm{dl}^{-1}\right)$ & $17,50 \pm 14,04$ & $17,82 \pm 18,71$ & $45,47 \pm 13,87$ & $35,58 \pm 13,69$ \\
\hline $\operatorname{ALT}\left(U / L^{-1}\right)$ & $104,05 \pm 62,93$ & $100,51 \pm 33,77$ & $74,82 \pm 40,89$ & $64,82 \pm 43,72$ \\
\hline $\operatorname{AST}\left(U / L^{-1}\right)$ & $187,04 \pm 102,01$ & $223,72 \pm 146,27$ & $177,89 \pm 52,75$ & $140,65 \pm 38,83$ \\
\hline URÉlA(mg/dl-1) & $49,57 \pm 11,18$ & $49,97 \pm 11,12$ & $40,20 \pm 9,50$ & $40,38 \pm 7,60$ \\
\hline CREATININA(mg/dL-1) & $0,52 \pm 0,10$ & $0,74 \pm 0,18$ & $0,57 \pm 0,19$ & $0,61 \pm 0,20$ \\
\hline
\end{tabular}

Em relação aos valores de Glicose, observa-se que os machos de 200 a 370 $\mathrm{g}$ de peso apresentaram valores maiores $\left(245,13 \mathrm{mg} / \mathrm{dl}^{-1}\right)$ que o outro grupo de ma- chos e fêmeas. Alguns trabalhos semelhantes 12,1 também constataram maior média de Glicose para animais machos. Lima ${ }^{2}$, trabalhando com ratos wistar, provenien- 
tes do Biotério da Universidade Tiradentes, também observa maiores médias de Glicose para os animais machos $\left(138,72 \mathrm{mg} / \mathrm{dl}^{-1}\right)$.

Com relação aos valores de glicose das fêmeas, observou-se no presente estudo, médias mais elevadas (170,38 e 166,07 $\mathrm{mg} / \mathrm{dl}^{-1}$ ) quando comparadas a outros estu$\operatorname{dos} 2,8,1$.

A glicose, assim como os outros parâmetros bioquímicos, é um parâmetro extremamente influenciado por muitos fatores. Tal como acontece com todos os mamíferos, a glicose no sangue, depende do tipo de alimentos consumidos e do tempo desde a última refeição. Quando o animal está em jejum, os níveis de glicose no sangue diminuem significativamente ao longo do tempo, uma vez que açúcares não estão sendo consumidos e metabolizados. Porém, se as taxas de glicose permanecem elevadas no rato em jejum, a Diabetes pode estar presente ou pode ocorrer ainda, que, se o animal é submetido a um período prolongado de jejum, o processo conhecido como gliconeogênese irá também elevar os níveis de glicose sanguínea.

Para os valores de triglicerídeos houve, neste estudo, uma variação de 64,49 a $46,73 \mathrm{mg} / \mathrm{dl}^{-1}$ para os machos e 78,68 a $95,10 \mathrm{mg} / \mathrm{dl}^{-1}$ para as fêmeas. Já para as médias de Colesterol total, observa-se uma variação de 54,69 a $44,96 \mathrm{mg} / \mathrm{dl}^{-1}$ para os machos e 69,69 a $71,85 \mathrm{mg} / \mathrm{dl}^{-1}$ para as fêmeas. Quando se verifica a literatura, observam-se dados bastante divergentes entre si para estes dois parâmetros 2,8,1,12. Lima², trabalhando com ratos machos e fêmeas de 140 a 310 g registrou médias de Colesterol para os machos de $60,68 \pm 6,51 \mathrm{mg} / \mathrm{dL}$ e de $64,86 \pm 11,17 \mathrm{mg} / \mathrm{dL}$ para as fêmeas, o que diverge dos dados obtidos na presente pesquisa.

Santo 201613 registra médias de colesterol total de $64,18 \mathrm{mg} / \mathrm{dL}$, para ratos de 60 gramas, alimentados com dietas ricas em óleos vegetais e nos quais a coleta de sangue foi realizada via punção cardíaca.
Em relação às médias de HDL, constatam-se médias de 17,50 e 17,82 mg/dl -1 para os machos de 200 a 370 g e 380 a 500 g respectivamente. Nas fêmeas foram observadas médias de $45,47 \mathrm{mg} / \mathrm{dl}^{-1}$ para as fêmeas de 150 a $230 \mathrm{~g}$ e $35,58 \mathrm{mg} / \mathrm{dl}^{-1}$ para as fêmeas de 230 a 300 g. Observa-se que para os machos, independente do peso, as médias de HDL se mantiveram na mesma faixa de valores. Já para as fêmeas, os níveis de HDL foram menores, para os animais mais pesados (230 a $300 \mathrm{~g}$ ).

O HDL é o maior no tamanho de partícula, em comparação às demais frações de colesterol, e está associado a um menor risco de aparecimentos de doenças como a doença arterial coronariana14. Através do transporte reverso do colesterol, o HDL leva os depósitos de colesterol arterial para serem processados no fígado, onde são convertidos em metabólicos úteis e, eventualmente, eliminados através das secreções biliares 15 . Além disso, segundo Gordon ${ }^{16}$, o HDL atua na função imunológica, inflamação vascular, metabolismo de glicose, e no processo de coagulação.

Com relação aos valores das enzimas hepáticas ALT e AST, observa-se na tabela 1, que os valores obtidos na presente pesquisa foram superiores aos encontrados por $11,2,8$. Já Santo 13 , registrou algumas médias de AST com valores semelhantes ao presente estudo (222,4 U/L; 191,0 U/L; 144,0 $\mathrm{U} / \mathrm{L}$ ) porém, para animais machos com $60 \mathrm{~g}$ de peso.

Diniz e colaboradores ${ }^{12}$, trabalhando também com ratos wistar machos e fêmeas, porém com animais de dois a três meses de idade, observaram valores de AST e ALT bem menores (AST Machos: 78,2; AST Fêmeas: 79,3; ALT machos: 59,0; ALT Fêmeas: 52,0) do que os apresentados no presente trabalho.

Nota-se, e se faz de extrema importância ressaltar, que os diferentes métodos utilizados para a realização de testes enzimáticos variam muito, fornecendo valores muitas vezes discrepantes. Por isso, a 
comparação com outros estudos, mesmo quando a unidade utilizada for a mesma, só será válida, se os mesmos substratos, $\mathrm{pH}$ e temperatura de reação forem utilizados.

De acordo com Haldelman e Blue ${ }^{17}$, a metodologia utilizada sempre interfere com os resultados laboratoriais. Lillie e colaboradores ${ }^{18}$ relatam ainda que os resultados só podem ser generalizados em determinadas condições específicas de estudo, com a mesma linhagem de ratos, idade e dieta, assim como a metodologia, que poderiam interferir nos resultados.

No que diz respeito ao perfil renal dos animais, observa-se na tabela 1, valores de ureia e creatinina, dentro da faixa de valores encontrados em outros estudos 8,12,2. Lock e colaboradores ${ }^{19}$, trabalhando com ratos wistar machos de $220 \mathrm{~g}$ de peso, observaram médias de ureia de $51,51 \mathrm{mg} \cdot \mathrm{dL}^{-1}$ e creatinina de $0,68 \mathrm{mg} \cdot \mathrm{dL}^{-1}$.

De acordo com os valores obtidos na presente pesquisa, verifica-se que entre os diversos parâmetros bioquímicos analisados, alguns parâmetros apresentaram similaridade com os valores encontrados em outros estudos, porém para outros parâmetros, os valores obtidos foram discordantes aos da literatura.

Dantas e colaboradores ${ }^{11}$ afirmam, ao analisar comparativamente os seus resultados com os apresentados na literatura, que ocorrem claramente, variações intraespécies, sendo provavelmente resultantes de diferenças de ordem ambiental, bem como de manuseio e/ou metodologia empregada. Estes autores ainda afirmam que é imprescindível que essas variações

\section{CONSIDERAÇÕES FINAIS}

Os resultados deste estudo caracterizam as condições fisiológicas de ratos wistar, mantidos no biotério das Faculdades Nova Esperança - PB e podem ser utilizados como referência na perspectiva de se traçar um perfil bioquímico de ratos wistar não tratados e acondicionados em sejam consideradas durante a pré-seleção dos animais, na avaliação e observação dos resultados obtidos nos procedimentos experimentais, assim como na análise das modificações induzidas por processos patológicos.

Vários foram os estudos 1,2,11,8,10 que utilizaram uma metodologia semelhante, com algumas poucas diferenças, para obter e analisar os parâmetros bioquímicos dos ratos, o que sugeriria que os valores bioquímicos destes trabalhos fossem semelhantes aos do presente estudo. Porém, não é o que ocorre, verificam-se várias diferenças nos valores observados. O que corrobora a necessidade gritante de se determinar os valores de referência específicos para cada biotério.

Os resultados são dados preliminares de uma caracterização fisiológica ebioquímica de ratos wistar, provenientes do Biotério das Faculdades Nova Esperança, comumente utilizados para fins experimentais distintos. Eles podem ser úteis para o grupo controle em determinados experimentos, mas não excluem novas determinações cada vez que animais sejam mantidos em condições experimentais particulares. Portanto, mais estudos são necessários para um melhor conhecimento da fisiologia animal.

Se faz de extrema importância também, o conhecimento e a divulgação dos valores dos parâmetros fisiológicos dos animais de experimentação, considerando que podem exibir variações influenciadas por vários fatores que devem ser levados em consideração nas pesquisas experimentais.

condições semelhantes.

A presente pesquisa também corrobora que vários fatores intrínsecos e extrínsecos podem promover a variação em alguns parâmetros e, portanto, cada centro de pesquisa deve ter seus valores fisiológicos estabelecidos. 


\title{
REFERENCE VALUES FOR BIOCHEMICAL PARAMETERS OF UNTREATED WISTAR RATS WHITH DIFERENT WEIGHT RANGES FROM THE JOÃO BEZERRA DE LIMA BIOTERIUM AT NOVA ESPERANÇA COLLEGE IN PARAÍBA
}

\begin{abstract}
Rodents are biological systems sensitive to external and internal factors, so that intraspecies variations are evident in the experimental results, being certainly products of environmental differences, handling and/ or methodology adopted. Therefore, it is essential to establish proper reference values for each bioterium or laboratory, as well as to disseminate them. The objective of this study was to establish reference values for biochemical parameters of untreated Wistar rats from the João Bezerra de Lima Bioterium at Nova Esperança College in Paraíba state, Brazil. Blood samples were collected from 83 Wistar rats, male and female, adult, healthy, different generations, weighing 200 to $500 \mathrm{~g}$ for males and 150 to $300 \mathrm{~g}$ for females, from the João Bezerra de Lima Bioterium, Nova Esperança College in Paraíba State. The biochemical parameters were glucose, triglycerides, total cholesterol, HDL, AST and ALT enzymes and urea and creatinine. The study was evaluated by CEUA FACENE / FAMENE and approved, under protocol number 0051.2017.1. The averages of some of the biochemical parameters recorded are as follows: Glucose (Males from 200 to $370 \mathrm{~g}-245,13 \mathrm{mg} / \mathrm{dL}-1$; males from 380 to $500 \mathrm{~g}-212,39 \mathrm{mg} / \mathrm{dL}-1$; females from 150 to $230 \mathrm{~g}-170,38 \mathrm{mg} / \mathrm{dL}-1$; Females from 230 to $300 \mathrm{~g}$ - 166,07 mg/dL-1); Colesterol total: (Males from 200 to $370 \mathrm{~g}-54,69 \mathrm{mg} / \mathrm{dL}-1$; males 380 to $500 \mathrm{~g}$ - 44,96 $\mathrm{mg} / \mathrm{dL}-1$; Females from 150 to $230 \mathrm{~g}$ - 69,69 mg/dL-1; Females from 230 to $300 \mathrm{~g}-71,85 \mathrm{mg} / \mathrm{dL}-1$ ); Urea:(Males from 200 to $370 \mathrm{~g}-49,57 \mathrm{mg} / \mathrm{dL}-1$; males 380 to $500 \mathrm{~g}$ - 49,97 mg/dL-1; Females from 150 to 230g - 40,20 mg/ $\mathrm{dL}-1$; Females from 230 to $300 \mathrm{~g}-40,38 \mathrm{mg} / \mathrm{dL}-1)$. The results characterize the physiological conditions of the animals kept in the Bioterium of Nova Esperança College in Paraíba state. Many intrinsic and extrinsic factors may promote variation of the parameters, so each research center must have its physiological values established.
\end{abstract}

\section{KEYWORDS}

Biochemistry. Bioterium. Rats. Reference Values. Wistar.

\section{REFERÊNCIAS}

1 Castello Branco A, Diniz M, Almeida R, Santos H, Oliveira K, Ramalho J, et al. Parâmetros Bioquímicos e Hematológicos de Ratos Wistar e Camundongos Swiss do Biotério Professor Thomas George. Revista Brasileira de Ciências da Saúde. 2011; 15(2): 209-214.

2 Lima C, Lima A, Melo M, Dória G, Leite $B$, Serafini $M$, et al. Valores de referência hematológicos e bioquímicos de ratos (Rattus novergicus linhagem Wistar) provenientes do biotério da Universidade Ti- radentes. Scientia Plena. 2014;10(3).

3 Harkness S, Wagner J. Biologia e Clínica de coelhos e roedores. 3rd ed. São Paulo: Livraria Roca Ltda.; 1993.

4 Fagundes D, Taha M. Modelo animal de doença: critério de escolha e espécies de animais de uso corrente. Acta Cir Bras. 2004;19.

5 Cooper A, Johnson C. Animal experimentation. Br J Surg. 1991;78:1409-11. 
6 Nunes D, Favali C, Souza-Filho A, Silva A, Filgueiras T, Lima M. Evaluation of cellular profile and main constituents the rat and mouse blood from the animal house of the Federal University of Ceará,Brazil. Rev. Med. UFC. 2004; 34 (1-2):21-29.

7 Pinheiro D, Favali C, Filho A, Silva A, Filgeuiras T, Lima M. Parâmetros Hematológicos de Camundongos e Ratos do Biotério Central da Universidade Federal do Ceará. Bol. Inf. Cobea. 1997/98; (3): 6-9.

8 Melo M, Dória G, Serafini M, Araújo A. Valores de referência Hematológicos e Bioquímicos de Ratos (Rattus novergicus linhagem Wistar) provenientes do biotério central da Universidade Federal de Sergipe. Scientia Plena. 2012; 8, 049903.

9 Bruno G, Menezes V, Bruno J, Almeida M, Viana G. Avaliações hematológicas e bioquímicas do sangue de cães submetidos à pulpotomias com cimento de antibiótico. Revista de Odontologia da UNESP. 2006; 35(3): 125-133.

10 Carvalho G, Masseno A, Zanini M, Zanini S, Porfírio L, Machado J, et al. Avaliação clínica de ratos de laboratório (Rattus novergicus linhagem Wistar): parâmetros sanitários, biológicos e fisiológicos. Revista Ceres. 2009; 56(1): 051-057.

11 Dantas J, Ambiel , Cuman R, Baroni S, Bersani-Amado $C$. Valores de referência de alguns parâmetros fisiológicos de ratos do Biotério Central da Universidade Estadual de Maringá, Estado do Paraná. Acta Scientiarum. Health Science. 2006; 28(2):165-170.

12 Diniz M, Medeiros I, Santos H, Oliveira $\mathrm{K}$, Vasconcelos T, Aguiar F, et al. Padroni- zação dos Parâmetros Hematológicos e Bioquímicos de Camundongos Swiss e Ratos Wistar. Revista Brasileira de Ciências da Saúde. 2006; 10(2): 171-176.

13 Santo B, Figueiredo P, Silva C, Filiú W, Conceiçon A, Santos E, et al. Impacto de diferentes fontes lipídicas em parâmetros bioquímicos de ratos wistar. Revista UNIABEU Belford Roxo. 2016: 9(23): 140-148.

14 Chatterjee C, Sparks D. Hepatic Lipase, High Density Lipoproteins, and Hypertriglyceridemia. American Journal of Pathology. 2011: 178(4): 1429-33.

15 Yasuda T, Ishida T, Rader D. Update on the Role of Endothelial Lipase in High-Density Lipoprotein Metabolism, Reverse Cholesterol Transport, and Atherosclerosis. Circulation Journal. 2010: 74 (11): 2263-70.

16 Gordon S, Hofmann S, Askew D, Davidson W. High density lipoprotein:it's not just about lipid transport anymore.. Trends Endocrinology and Metabolism.. 2011: 22(1): 9-15.

17 Haldelman C, Blue J. Laboratory data: read beyond the numbers. In Veterinary laboratory medicine:in practice.. Trenton: Veterinary Learning Systems; 1993.

18 Lillie L. et al. Reference values for young normal Sprague-Dawley rats: weight gain, hematology and clinical chemistry. Hum. Exp. Toxicol. 1996: 15 (8): 612-616.

19 Lock F, Valduga A, Macedo S, Cichoski A. Perfil bioquímico de ratos alimentados com iogurte contendo extrato de erva-mate (Ilex paraguariensis St. Hil). Braz. J. Food Technol. 2011: 14(4): 332-337. 